$\mathrm{M}$ етотрексат: к вопросу об эффективности и безопасности применения препарата у больных псориазом

А.Л. Бакулев

ФГБОУ ВО «Саратовский государственный медицинский университет им. В.И. Разумовского» Минздрава России 410012, г. Саратов, ул. Большая Казачья, д. 112

\footnotetext{
Приводятся современные данные литературы по метотрексату, который в настоящее время является одним из наиболее часто используемых препаратов для системной терапии больных среднетяжелым и тяжелым псориазом. Обсуждаются вопросы оценки специалистами ответа на системную терапию псориаза и возможные терапевтические стратегии; выбора инициирующих доз метотрексата в лечении пациентов с псориазом; возможностей совместного применения с генно-инженерными биологическими препаратами; мониторирования терапии.

Приводятся данные рандомизированных клинических исследований по долгосрочному непрерывному лечению метотрексатом (эфффективность, безопасность); о способах его введения в организм пациентов; сроках и критериях долгосрочной эфрфективности.

Представлены сведения о механизмах действия метотрексата и новые данные о влиянии на обмен аденозина и способности препарата модулировать воспалительный ответ в коже у пациентов путем ингибирования клеточных компонентов воспалительного инсрильтрата в коже (дендритные антигенпродуцирующие клетки и Т-лимсоциты), а также о подавлении экспрессии ряда провоспалительных цитокинов (IFN- $\gamma$ и IL-17A).
}

Ключевые слова: псориаз, системная терапия, метотрексат, эффективность, безопасность, ответ на терапию, обмен фолатов, аденозин, воспаление.

Контактная инсормация: misha.juchkov@gmail.com. Вестник дерматологии и венерологии 2017; (1): 38—45.

\title{
Methotrexate: Revisited efficiency and safety of drug administration in psoriasis patients
}

\section{A. L. Bakulev}

Saratov State Medical University named after V.I. Razumovsky Bolshaya Kazachiya str., 112, Saratov, 410012, Russia

The article presents the current data of the literature on methotrexate, which is now one of the most commonly used preparation for the systemic treatment of patients with moderate to severe psoriasis.

The following problems are under consideration: estimation by specialists of response to systemic psoriasis therapy and possible therapeutic strategies; selecting initial doses of methotrexate for the treatment of patients with psoriasis; the possibilities of combined use with genetically engineered biological agents and monitoring of therapy.

The data from randomized clinical trials on the long-term continuous treatment with methotrexate (efficacy, safety); methods of its administration to patients and time and criteria for long-term effecasy are reported.

There are presented the data on the mechanisms of methotrexate action and the new data about the impact on the adenosine metabolism and the ability of the preparation to modulate the inflammatory response in the skin of patients by inhibiting the cellular components of the inflammatory infiltrate in the skin (dendritic antigen-producing cells and T-lymphocytes), as well as the suppression of expression of some proinflammatory cytokines (IFN- $\gamma$ and IL17A).

Key words: psoriasis, systemic therapy, methotrexate, efficacy, safety, response to therapy, pholate metabolism, adenosine, inflammation.

Corresponding author: misha.juchkov@gmail.com. Vestnik Dermatologii i Venerologii 2017; 1: 38—45. 
Псориаз - иммуновоспалительное заболевание, характерными особенностями которого являются гиперпролиферация кератиноцитов, нарушение их диффференцировки, воспалительная инфильтрация и неоангиогенез в коже. Данный дерматоз имеет хроническое течение и в настоящее время рассматривается в одном ряду с такими патологиями, как ревматоидный артрит и неспецифические воспалительные заболевания кишечника, как с точки зрения неблагоприятного влияния на качество жизни пациентов, так и в связи с необходимостью осуществления длительного контроля над его течением, препятствования развитию и прогрессированию псориатического артрита, а также сопутствующих коморбидных состояний, патогенетически взаимосвязанных с псориазом [1-3].

В терапии больных среднетяжелым и тяжелым псориазом в настоящее время применяются как широко известные терапевтические методы и средства, в том числе фототерапия (фотохимиотерапия; узкополосная средневолновая УФ-терапия, эксимерный свет), метотрексат, циклоспорин А, ароматические ретиноиды (ацитретин), так и инновационные современные лекарственные препараты, к которым относятся таргетные синтетические базисные препараты, именуемые также селективными ингибиторами сигнальных путей (апремиласт, тофрацитиниб), и генно-инженерные биологические препараты (ремикейд, адалимумаб, этанерцепт, устекинумаб, секукинумаб). Вектор развития дерматовенерологии в плане разработки новых терапевтических возможностей для лечения пациентов, страдающих псориазом, в последние годы направлен именно на последние две группы лекарственных средств [1, 4-7].

Вместе с тем исследователям в современных условиях «бума» средних молекул и генно-инженерных биологических препаратов удалось уточнить ряд новых иммунопатогенетических особенностей псориаза и посмотреть на традиционно используемые медикаментозные препараты, в частности метотрексат, с совершенно другой стороны. Именно анализу данных последних лет по вопросам эфффективности, безопасности метотрексата при псориазе, а также принципиально новым сведениям о механизме терапевтического действия данного терапевтического средства посвящен настоящий обзор литературы.

Метотрексат применяется для лечения больных псориазом более 50 лет. За эти годы специалисты накопили огромный опыт практического применения данного лекарственного средства в условиях реальной клинической практики. Последнее обстоятельство, а также мощное цитостатическое действие позволили метотрексату в настоящее время стать терапевтическим средством первой линии в лечении пациентов с данным дерматозом и своего рода эталоном, с которым принято сравнивать эффрективность и безопасность новых терапевтических средств/методов, пред- лагаемых для лечения псориаза. Препарат демонстрирует высокую терапевтическую эфрфективность не только при вульгарной фрорме псориаза, но и при таких сложных с точки зрения терапии больных формах дерматоза, как псориатическая эритродермия и генерализованный пустулезный псориаз. Также несомненным достоинством метотрексата является его способность оказывать терапевтическое действие В отношении клинических проявлений псориатического артрита, который имеется примерно у 1/3 лиц, страдающих псориазом [8-10].

Несмотря на обилие публикаций по данной тематике, ряд проблем, связанных с применением метотрексата при псориазе, в настоящее время нельзя считать окончательно решенными. В частности, определенный интерес для специалистов представляют такие вопросы, как оценка ответа на терапию (выявление неответчиков); выбор инициирующих доз терапии; длительное непрерывное лечение данным препаратом; способ введения метотрексата в организм пациентов; сроки и критерии долгосрочной эфффективности, а также проблемы безопасности длительного применения препарата.

\section{Оценка ответа на терапию}

Вопросы оценки клинической эфффективности проводимой системной терапии псориаза у пациентов в настоящее время считаются достаточно разработанными. Общепринято, что при ответе на терапию по индексу PASI на 75\% и более (PASI 75) последняя считается успешной и должна быть продолжена.

В случае если удается достичь редукции тяжести и распространенности процесса на коже менее чем на 50\% (PASI 50), целесообразно принять решение о пересмотре терапевтической стратегии (уменьшить интервалы между приемом/введением лекарственного средства; увеличить дозу применяемого лекарственного средства; использовать комбинацию лекарственных средств; произвести смену лекарственного средства). Так, при возникновении «френомена ускользания терапевтического эффекта», описанного у генно-инженерных биологических препаратов из группы анти-TNF- $\alpha$, дополнительное назначение метотрексата является патогенетически оправданным из-за способности препарата достоверно уменьшить образование нейтрализующих аутоантител к биологическому агенту, что позволяет в конечном счете продолжить проводимую биологическую терапию. С другой стороны, очевидно, что пересмотр схемы лечения может ухудшить профиль «польза/риск» и стать причиной возникновения более частых нежелательных явлений терапии.

Наконец, при терапевтическом ответе по индексу PASI от 51 до 74\% вопрос о продолжении или смене терапевтической тактики должен решаться индивидуально с учетом таких фракторов, как локализация 
псориатических эфрфлоресценций и влияние псориаза на качество жизни конкретного больного. При наличии псориатических элементов сыпи в проблемных зонах (волосистая часть головы, лицо, ладони/подошвы, складки, гениталии/перианальная область), а также при отсутствии динамики по индексу качества жизни DLQI на 5 или более пунктов по сравнению с исходным уровнем следует также изменить терапевтическую стратегию по принципам, описанным выше. В других случаях проводимая системная терапия может быть продолжена и/или усилена за счет применения более мощных топических средств, в том числе содержащих фиксированные комбинации активных компонентов.

Сроки оценки ответа на терапию метотрексатом существенно отличаются от принятых для генно-инженерных биологических средств. При использовании последних первичную оценку их эффективности принято проводить в сроки от 16 до 22 нед. непрерывной терапии. Оценить ответ, а также установить первичных неответчиков (критерий - PASI 50) на терапию метотрексатом возможно уже через 8 нед. лечения данным препаратом [11-13].

\section{Подбор инициирующей дозы метотрексата}

Вопрос о дозах метотрексата, которые следует применять в начале курса лечения псориаза у больных, остается весьма актуальным. В международных и отечественных клинических рекомендациях по ведению пациентов с данным дерматозом единого мнения в данном вопросе нет. Так, Европейская академия дерматовенерологии в своих рекомендациях отмечает, что первоначальная доза метотрексата для лечения больных псориазом должна составлять от 7,5 до 15 мг препарата в неделю. Далее доза может быть увеличена в зависимости от эфффективности у конкретного пациента, однако максимальная доза не должна превышать 22,5 мг в неделю $[14,15]$. В клинических рекомендациях в Германии подчеркивается, что начинать системную терапию метотрексатом целесообразно сразу с большей дозы (15 мг в неделю); терапию в дозе 15 мг препарата в неделю следует продолжать в течение 8 нед. и в случае отсутствия улучшения со стороны процесса на коже с 9-й недели лечения дозу метотрексата следует увеличить до 20-25 мг в неделю. Более того, немецкие специалисты отмечают, что инициирующая терапия меньшими дозами с последующим их увеличением не является целесообразной из-за отсутствия преимуществ как по эффективности, так и по безопасности [16]. G. Carretero и соавт. (2010) при формулировке клинических рекомендаций Испанской академии дерматовенерологии подчеркивают, что низкие дозы метотрексата в качестве инициирующей терапии следует назначать только пациентам, которым данный лекарственный препарат назначается впервые [17]. При наличии указаний в анамнезе на ранее успешное применение данного лекарственного средства у конкретного пациента стартовая доза препарата может варьировать от 15 до 30 мг в неделю. В клинических рекомендациях NICE (Великобритания, 2012) по ведению пациентов с псориазом даются следующие рекомендации специалистам по выбору стартовых дозировок метотрексата для лечения больных псориазом целесообразно использовать 5-10 мг вышеназванного препарата один раз в неделю и затем постепенно увеличивать дозу до минимально эффрективной, но не более 25 мг в неделю [18]. Наконец, в клинических рекомендациях Российского общества дерматовенерологов и косметологов (2015) указывается, что при приеме внутрь метотрексат назначается по 5-7,5-10-15 мг в неделю однократно. Начальная доза метотрексата при парентеральном применении составляет 7,5-10 мг в неделю, при необходимости она может быть увеличена до 30 мг в неделю. После достижения терапевтического эфффекта возможна поддерживающая терапия в минимальной эффрективной дозе (однако не более 22,5 мг в неделю) [19].

Таким образом, можно констатировать, что единого подхода к выбору дозировок для инициирующей терапии метотрексатом в настоящее время не существует. Более того, при назначении препарата специалистам следует учитывать такие факторы, как тяжесть и распространенность псориаза; наличие и активность псориатического артрита (при его наличии); профиль самого пациента (масса тела; сопутствующие патологии; результаты гематологического, биохимического анализов крови и т.д.). Определенную роль, вероятно, также могут играть характеристики самого метотрексата, выпускаемого разными фрирмами-производителями и отличающегося по способам введения в организм и, соответственно, биодоступности. Вместе с тем общность в подходах к назначению метотрексата сводится к следующему:

первоначальная доза должна быть меньше терапевтически эффективной, что прежде всего продиктовано вопросами безопасности терапии;

у лиц, ранее получавших препарат, начальная доза может быть большей в сравнении с пациентами, которым препарат назначается впервые;

постепенно дозу препарата следует увеличить до минимально приемлемой по профилю «польза/ риск».

В настоящее время является общепринятым, что ключевую роль в формулировании рекомендаций относительно терапии тех или иных заболеваний играют результаты рандомизированных клинических исследований, выполненных в соответствии с правилами надлежащей клинической практики GCP. В последние годы были опубликованы результаты трех независимых друг от друга клинических исследований, из которых можно получить данные по возможным стартовым дозам метотрексата. В данных исследованиях метотрексат тестировали у больных среднетяжелым и тяжелым псо- 
риазом в сравнении с генно-инженерными биологическими препаратами - бриакинумабом* (исследование M10-255), инорликсимабом (исследование RESTORE) и адалимумабом (исследование CHAMPION), с чем связан тот фракт, что оценка эффрективности терапии проводилась в сроки, принятые для генно-инженерных биологических препаратов [20-22]. Следует отметить, что дизайн всех трех вышеназванных клинических исследований предполагал применение пероральной формы метотрексата. Начальные дозы препарата в вышеназванных исследованиях существенно варьировали - от 5 до 15 мг в неделю (см. таблицу). Несмотря на это, результаты всех трех протоколов оказались вполне сопоставимы между собой по показателю ответа (PASI 75) на инициирующую терапию данным лекарственным препаратом, который в исследовании m10-255 составил 39,9\% (на 24-й неделе терапии); в исследовании RESTORE - 41,9\% (на 16-й неделе непрерывного лечения пациентов метотрексатом) и в исследовании CHAMPION - 35,5\% на 16-й неделе такой терапии (прямые сравнения ответа по PASI 75 на инициирующую терапию метотрексатом у больных псориазом к настоящему времени не проводились). Таким образом, можно сделать вывод, что инициирующая доза метотрексата не является единственным и ключевым фактором эффективности терапии больных среднетяжелыми и тяжелыми формами псориаза.

\section{Длительное непрерывное лечение метотрексатом: вопросы эффективности и безопасности}

Специалисты-дерматологи длительное время использовали курсовое лечение метотрексатом исключительно во время обострения дерматоза с последующей его отменой. Такая стратегия применения препарата считалась относительно безопасной с точки зрения возможного риска развития серьезных нежелательных явлений терапии в отдаленной перспективе, особенно таких грозных, как фиброз или цирроз печени [23]. Вместе с тем врачи разных специальностей в последние годы весьма часто стали использовать длительные схемы назначения метотрексата для контроля самых различных заболеваний. В частности, при псориатическом артрите метотрексат рассматривается в качестве основного базисного противовоспалительного средства, позволяющего в определенной степени контролировать такие его проявления, как периферический артрит и дактилит; применяется длительными курсами [24, 25]. Считается, что малые дозы данного лекарственного препарата при длительном применении не позволяют добиться адекватного клинического ответа и контроля над течением псориатического артрита.

\footnotetext{
* В настоящее время бриакинумаб не зарегистрирован в России для лечения больных псориазом.
}

В дерматологии ранее считалось, что ключевым фрактором, определяющим безопасность длительного применения метотрексата, является суммарная доза препарата, полученная пациентом в течение жизни (1500 мг), а биопсия печени рассматривалась в качестве референсного теста, который позволял ответить на вопрос о возможности/невозможности продолжения терапии данным препаратом в условиях набора суммарной дозы. В настоящее время вышеназванные подходы пересмотрены. С современных позиций риск гепатотоксичности определяется по данным первоначальных результатов и анализа тенденций с течением времени таких лабораторных показателей функции печени, как аланин-, аспартатаминотрансфераза, гаммаглутамилтранспептидаза с обязательным определением содержания проколлагена III типа в сыворотке крови с учетом существующих и потенциальных факторов риска (ожирение, сахарный диабет и употребление алкоголя). При анализе результатов сывороточного содержания проколлагена III типа следует помнить, что данный тест не может быть использован у детей и подростков; результаты могут быть некорректными у людей с псориатическим артритом; прогностическая ценность положительного результата вариабельна (23-95\%), а отрицательного — весьма высока (89$100 \%$ ) [18].

R. Warren и соавт. (2016) опубликовали результаты 52-недельного двойного слепого рандомизированного, плацебо-контролируемого исследования МЕТОР (METOthrexate in patients with moderate-to-severe plaque type Psoriasis), в котором подробно проанализированы данные по долгосрочной эффективности и безопасности метотрексата у взрослых пациентов со среднетяжелыми и тяжелыми формами вульгарного псориаза [26]. Исследование проводилось в 16 независимых друг от друга центрах, расположенных в Германии, Франции, Нидерландах и Великобритании, и в нем приняли участие 120 пациентов.

Дизайн исследования МЕТОР предполагал рандомизацию пациентов на две группы на 0-й неделе после стандартных процедур скрининга участников. Пациенты 1-й группы в течение всего исследования получали терапию метотрексатом. При этом пациентам 2-й группы на 0-16-й неделе назначался препарат-плацебо. В исследовании использовали метотрексат для подкожного введения в предварительно заполненных шприцах (производитель - компания «Медак ГмбХ», Германия), зарегистрированный (в том числе в России) под коммерческим названием Методжект ${ }^{\circledR}$. Выбор лекарственного препарата и способа его введения в организм в данном протоколе был обусловлен низким риском случайной передозировки и более высоким содержанием активного метаболита метотрексата (полиглутаминированной формы) при его парентеральном введении. Режим дозирования метотрексата в соответствии 
с протоколом предполагал постепенную эскалацию дозы. Пациентам 1-й группы на 0-16-й неделе исследования вводили подкожно 17,5 мг метотрексата один раз в неделю (у лиц, которые не достигали PASI 50 через 8 нед. от начала терапии, дозу метотрексата увеличивали до 22,5 мг). На 17-52-й неделе участия в данном протоколе пациенты 1-й группы продолжали получать вышеназванные дозы метотрексата. Если участнику исследования, получавшему метотрексат в дозе 17,5 мг, на 24-й неделе не удавалось достигнуть значений PASI 75, то ему увеличивали дозу до 22,5 мг. В случае если доброволец уже получал дозу 22,5 мг метотрексата в неделю и на 24-й неделе не демонстрировал ответ на терапию выше чем PASI 50, он исключался из исследования.

Пациенты 2-й группы получали инъекции-плацебо на 0-16-й неделе участия в исследовании, а затем переводились на метотрексат для подкожного введения в дозе 17,5 мг в неделю до окончания протокола с возможностью дальнейшего увеличения дозы до 22,5 мг на 24-й неделе при недостаточном ответе (оценка по PASI 50). В случае достижения терапевтического ответа PASI 75 на 16-й неделе лечения препаратом-плацебо пациенты данной группы далее вообще не получали каких-либо инъекций, а при рецидиве переводились на введение метотрексата подкожно в дозе 17,5 мг в неделю.

В соответствии с протоколом исследования все участники, получавшие метотрексат, через 24 ч после его подкожного введения принимали внутрь 5 мг фолиевой кислоты однократно.

Кроме оценки тяжести и распространенности псориатического процесса протоколом клинического исследования была предусмотрена динамическая оценка качества жизни (DLQI).

После 16 нед. терапии у пациентов 1-й группы ответ PASI 50 был достигнут у 66\% участников исследования; PASI 75 - у 41\%; PASI 90 - у 18\%. Во 2-й группе через 16 нед. участия в данном исследовании ответ PASI 50 был достигнут у 31\% пациентов; PASI 75 у 10\%; PASI 90 - у 0\%. Обращает на себя внимание тот факт, что в 27\% наблюдений у больных 1-й группы PASI 75 был достигнут уже на 8-й неделе терапии метотрексатом, вводимым подкожно.

Результаты оценки тяжести и распространенности псориаза на 52-й неделе исследования следующие: в 1-й группе снижение индекса PASI 50 констатировали в $58 \%$ случаев, PASI 75 - в $45 \%$, PASI 90 - в 27\%; во 2-й группе - соответственно в 34, 34, $28 \%$.

Снижение индекса DLQI до значений $\leq 5$, что соответствовало слабому влиянию заболевания на качество жизни, отметили на 16-й неделе у $59 \%$ лиц в 1-й группе и у 34\% - во 2-й группе. Данный показатель на 52-й неделе терапии составил соответственно 55 и $45 \%$.
У так называемых первичных неответчиков на терапию метотрексатом на 8-й неделе лечения дальнейшая эскалация дозы препарата способствовала повышению терапевтической эффективности предпринятой терапии, в то время как недостаточный эффрект по PASI на 24-й неделе (вторичные неответчики) требовал отмены курсовой терапии метотрексатом изза ее неэффрективности и невозможности дальнейшего увеличения дозы препарата до токсической.

Таким образом, результаты протокола МЕТОР свидетельствуют о том, что долгосрочное подкожное введение метотрексата позволяет добиться большего терапевтического ответа со стороны пациентов со среднетяжелым и тяжелым псориазом, чем его пероральное применение, что сопровождается уменьшением неблагоприятного влияния дерматоза на качество жизни больных (прямые сравнения метотрексата для приема внутрь и подкожного введения в настоящее время опубликованы только для лиц с псориатическим артритом). При длительном применении метотрексата для подкожного введения терапевтический эффрект развивается в более ранние сроки и остается стабильным в течение 52 нед. Недостаточный эффрект от проводимого лечения метотрексатом на 8-й неделе требует эскалации дозы препарата, в то время как на 24-й неделе свидетельствует о неэфрективности лечения и целесообразности пересмотра стратегии терапии у конкретного пациента.

При проведении долгосрочной терапии метотрексатом вопросы безопасности приобретают большую актуальность. Наиболее часто среди нежелательных явлений при длительном применении метотрексата (исследование МЕТОР) встречались различные инфекции (64\%), гастроинтестинальные нарушения (33\%), повышение уровня различных показателей функции печени (23\%), а также лейкопения (5\%). При этом у участников данного исследования случаев смерти, серьезных инфекционных заболеваний и злокачественных опухолей зарегистрировано не было.

В структуре нарушений со стороны желудочнокишечного тракта преобладали диспепсические растройства (22\%), в то время как диарея встречалась в 7\% наблюдений, боль в животе - в 3\%, эрозивные десекты в полости рта - в 3\%.

Транзиторное повышение уровня печеночных ферментов в 2 раза отмечали в 19\% наблюдений; в 3 раза и более- в 15\%. Вышеназванные изменения функциональных показателей печени были стойкими в $12 \%$ случаев, что стало причиной прекращения введения метотрексата и выбывания пациентов из исследования. При этом у всех наблюдавшихся лиц с патологическими изменениями тестированных показателей (аланин-, аспартатаминотрансфераза, гаммаглутамилтранспептидаза) увеличения сывороточного содержания проколлагена III типа отмечено не было. 
Лейкопения, как правило, протекала легко и носила транзиторный характер; лишь в 1\% случаев констатировали лейкопению третьей степени тяжести и в 1\% наблюдали перманентное ее течение.

Таким образом, полученные данные свидетельствуют о приемлемой безопасности долгосрочного применения метотрексата для подкожного введения у пациентов, страдающих среднетяжелым или тяжелым псориазом.

\section{Механизм действия и модуляция воспалительного ответа}

Исследования последних лет существенно обогатили наши представления о патогенезе псориаза. С современных позиций известно, что стимулированные различными фракторами дендритные антигенпродуцирующие клетки способны активировать Т-клетки, которые на такую активацию отвечают повышенной экспрессией генов, отвечающих за синтез интерлейкинов IL-12 и IL-23. В результате происходит пролиферация и дифференцировка Т-лимфоцитов на две субпопуляции - Th-1 и Th-17, которые, в свою очередь, экспрессируют ряд генов, ответственных за синтез и последующий выброс в ткани большого числа медиаторов воспаления: IL-2, интерферона- $\gamma$ (IFN- $\gamma)$, фактора некроза опухоли $\alpha$ (TNF-a), IL-21, IL-22 и IL-17A. Считается, что именно IL-17A является тем важнейшим с точки зрения патогенеза псориаза провоспалительным цитокином, который реализует свои эффекты путем стимуляции и гиперпролиферации кератиноцитов. Последние, действуя по принципу обратной связи, сами способствуют дальнейшему образованию в коже провоспалительных цитокинов (в том числе того же IL-17A) и хемокинов, что приводит к дальнейшей гиперпролиферации, нарушению диффреренцировки кератиноцитов в эпидермисе и в конечном счете к акантозу [27, 28].

Метотрексат в течение многих лет рассматривался исключительно в качестве лекарственного средства, оказывающего цитостатическое и антипролиферативное действие на кератиноциты в коже путем мощного антифолатного эфффекта.

Известно, что данный эфрфект метотрексата реализуется через его вмешательство в метаболизм фолиевой кислоты путем конкурентного ингибирования фермента дигидрофолатредуктазы. Это ингибирование блокирует образование дезокситимидилатовой кислоты, которая необходима для синтеза ДНК кератиноцитов эпидермиса. Однако связь метотрексата с ферментом дигидрофолатредуктазой является обратимой, что требует присутствия большого количества молекул лекарственного средства для надежного торможения синтеза ДНК кератиноцитов. В противном случае связь «ломается» и фермент восстанавливает свою активность. Понимание этого механизма действия метотрексата не только пред- ставляется важным с теоретической точки зрения, но и имеет значение для практики, так как тесно взаимосвязано с таким понятием, как биодоступность $[26,29]$. Вот почему в последние годы изучение форм метотрексата для парентерального введения перестало ограничиваться лишь обсуждением вопросов приемлемой переносимости такой терапии со стороны желудочно-кишечного тракта.

Кроме того, общеизвестно, что метотрексат ингибирует образование пуринового кольца инозиновой кислоты, предшественника всех пуриновых нуклеотидов ДНК и РНК [29].

Недавно впервые была высказана гипотеза о том, что механизм действия метотрексата может быть значительно масштабнее ранее изученного, так как расширились научные знания об активном метаболите метотрексата, которым является его полиглутаминированная форма (полиглутамат метотрексата). В частности, была доказана важнейшая роль последнего в стимуляции образования аденозина, оказывающего мощное эндогенное противовоспалительное действие, реализуемое через индукцию апоптоза активированных лимфоцитов или путем ингибирования активации этих клеток и экспрессии определенных генов, ответственных за синтез ряда провоспалительных цитокинов и/или молекул адгезии. Однако прямых доказательств, указывавших на то, что антифолатный эффрект метотрексата - яркая, но не единственная грань его механизма действия, при псориазе не было [30-34].

В обсуждаемом ранее в данном обзоре клиническом исследовании МЕТОР у 27 пациентов (из них 6 получали плацебо, 14 - метотрексат; 7 оказались первичными неответчиками на терапию метотрексатом) в динамике были исследованы парные биоптаты кожи (каждый диаметром 3 мм), взятые по краю псориатических эффрлоресценций на 0-й неделе [26]. Один из биоптатов фриксировали 4\% раствором фрормалина с буфером для последующего гистологического исследования; второй - в RNAlater-paстворе - для проведения иммуногистохимического анализа. В динамике отбор биоптатов был произведен повторно на 16-й неделе клинического исследования.

У пациентов с хорошим ответом на терапию метотрексатом (достижение показателя PASI 75) на 16-й неделе терапии имелось не просто статистически достоверное снижение уровня CD-3-позитивных клеток и CDIlc-положительных дендритных клеток, а нормализация их количества. У лиц в данной группе в результате проведенной терапии метотрексатом содержание M-PHK IL-17A и IFN- $\gamma$ также уменьшилось до значений, соответствующих нормальным величинам в контроле. Более того, установлено, что индивидуальные изменения уровня MPHK IL-17A находились в сильной корреляционной взаимосвязи с индивидуальным ответом пациента на терапию метотрексатом $(p<0,001)$. 
Таблица

Режимы повышения начальных доз метотрексата в клинических исследованиях М10-255; RESTORE; CHAMPION, мг/нед.

\begin{tabular}{|c|c|c|c|c|c|c|c|c|c|}
\hline \multirow[t]{2}{*}{ Исследование } & \multirow[t]{2}{*}{ Число участников } & \multicolumn{8}{|c|}{ Недели исследований } \\
\hline & & $0-1$ & $2-3$ & $4-7$ & 6 & $8-11$ & 10 & $12-15$ & 16 \\
\hline M10-255 & 163 & $5-10$ & 15 & & & & $20^{*}$ & & $25^{*}$ \\
\hline RESTORE & 215 & 15 & 15 & & 20 ** & & & & \\
\hline CHAMPION & 110 & 7,5 & 10 & 15 & & $20^{\#}$ & & $25^{\# \#}$ & \\
\hline
\end{tabular}

Примечания: * дозу повышали при PGA $\geq 2$ или PASI < 75; ** дозу повышали при изменениях PASI < 25 в сравнении с baseline-визитом; \# дозу повышали при PASI < 50; \#\# дозу повышали при PASI < 50 на 8-12 неделях клинического исследования.

Вышеперечисленные иммунологические эффректы у пациентов с хорошим ответом на терапию метотрексатом полностью совпали с динамикой снижения количества Кі-67-пролиферирующих кератиноцитов и микроабсцессов Мунро в эпидермисе. Таким образом, на современном уровне было доказано, что метотрексат способен модулировать воспалительный ответ в коже у больных псориазом. Препарат ингибирует ключевые клеточные компоненты воспалительного инфильтрата в коже (дендритные антигенпродуцирующие клетки и Т-лимсроциты), а также подавляет эксперссию таких важнейших провоспалительных цитокинов, как IFN- $\gamma$ и IL-17A. Последнее представляется особенно важным с учетом установленного в настоящий момент ключевого патогенетического значения IL-17A в стимуляции пролиферации кератиноцитов и нарушения их диффференцировки при псориазе [26, 35].

Данные о роли полиглутаминированной формы метотрексата в регуляции обмена аденозина на клеточ- ном уровне, а также результаты, полученные в рамках клинического исследования МЕТОР о противовоспалительных эффектах метотрексата, представляют большой научный интерес. Уровень развития медицинской науки в настоящее время, вероятно, позволит получить в ближайшее время новые данные об особенностях механизма модулирования воспалительного ответа в процессе терапии метотрексатом у больных псориазом.

В 2016 г. Всемирная организация здравоохранения в своем докладе подчеркнула, что псориаз представляет собой заболевание пожизненного характера и обратила внимание на необходимость разработки препаратов, доступных для лечения пациентов, страдающих данной патологией [36]. Лекарственный препарат метотрексат вполне соответствует данному требованию, при этом являясь весьма эфффективным терапевтическим средством с приемлемыми безопасностью и переносимостью.

\section{Литература}

1. Gottlieb A., Korman N. J., Gordon K. B. et al. Guidelines of care for the management of psoriasis and psoriatic arthritis: section. Psoriatic arthritis: overview and guidelines of care for treatment with an emphasis on the biologics. J Am Acad Dermatol 2008; 58: 851—64.

2. Augustin M., Reich K., Glaeske G. et al. M. Comorbidity and agerelated prevalence of psoriasis: analysis of health insurance data in Germany. Acta Derm Venereol 2010; 90: 147—51.

3. Gladman D. D., Antoni C., Mease P. et al. Psoriatic arthritis: epidemiology, clinical features, course, and outcome. Ann Rheum Dis 2005; 64 Suppl 2): ii14-7.

4. Baker C., Mack A., Cooper A. et al. Treatment goals for moderate to severe psoriasis: an Australian consensus. Australas J Dermatol 2013 May; 54 (2): $148-54$.
5. Daudén E., Puig L., Ferrándiz C. et al. Consensus document on the evaluation and treatment of moderate-to-severe psoriasis: Psoriasis Group of the Spanish Academy of Dermatology and Venereology. Spanish Psoriasis Group of the Spanish Academy of Dermatology and Venereology. J Eur Acad Dermatol Venereol 2016 Mar; 30 (Suppl 2): 1—18.

6. Sterry W., Barker J., Boehncke W. H. et al. Biological therapies in the systemic management of psoriasis: International Consensus Conference. Br J Dermatol 2004 Aug; 151 (Suppl 69): 3-17.

7. Thaçi $\mathrm{D}$. Long-term data in the treatment of psoriasis. Br J Dermatol 2008 Aug; 159 (Suppl 2): $18-24$.
8. Montaudié H., Sbidian E., Paul C. et al. Methotrexate in psoriasis: a systematic review of treatment modalities, incidence, risk factors and monitoring of liver toxicity. J Eur Acad Dermatol Venereol 2011 May; 25 (Suppl 2): 12-8.

9. Dogra S., Mahajan R. Systemic methotrexate therapy for psoriasis: past, present and future. Clin Exp Dermatol 2013 Aug; 38 (6): 573—88.

10. Yélamos 0., Puig L. Systemic methotrexate for the treatment of psoriasis. Expert Rev Clin Immunol 2015 May; 11 (5): 553-63.

11. Mrowietz U., Kragballe K., Reich K. et al. Definition of treatment goals for moderate to severe psoriasis: a European consensusArch Dermatol Res 2011 Jan; 303 (1): 1—10. 
12. Mrowietz U. Implementing treatment goals for successful long-term management of psoriasis.J Eur Acad Dermatol Venereol 2012 Mar; 26 (Suppl 2): 12-20.

13. Oji V., Luger T.A. The skin in psoriasis: assessment and challenges. Clin Exp Rheumatol 2015 Sep-0ct; 33 (5 Suppl 93): S14-9.

14. Nast A., Gisondi P., Ormerod A. D. et al. European S3-Guidelines on the systemic treatment of psoriasis vulgaris_Update 2015-Short version-EDF in cooperation with EADV and IPC. J Eur Acad Dermatol Venereol 2015 Dec; 29 (12): 2277-94.

15. Nast A., Jacobs A., Rosumeck S., Werner R. N. Methods Report: European S3-Guidelines on the systemic treatment of psoriasis vulgaris-update 2015-EDF in cooperation with EADV and IPC. J Eur Acad Dermatol Venereol 2015 Dec; 29 (12): e1-22.

16. Nast A., Boehncke W. H., Mrowietz U. et al. German S3-guidelines on the treatment of psoriasis vulgaris (short version). Arch Dermatol Res 2012 Mar; 304 (2): 87-113.

17. Carretero G., Puig L, Dehesa L. et al. Guidelines on the use of methotrexate in psoriasis. Actas Dermosifiliogr 2010 Sep; 101 (7): 600—13.

18. Smith C., Chandler D., Hepple P. et al. Psoriasis: assessment and management. NICE Clinical guideline. Published: 24 October 2012 nice. org.uk/guidance/cg153.

19. Псориаз. Федеральные клинические рекомендации. Дерматовенерология 2015: Болезни кожи. Инфекции, передаваемые половым путем. 5-е изд., перераб. и доп. М: Деловой экспресс 2016;. 415-470.

20. Reich K., Langley R. G., Papp K. A. et al. A 52-week trial comparing briakinumab with methotrexate in patients with psoriasis.N Engl J Med 2011 Oct 27;365 (17): 1586—96.
21. Barker J., Hoffmann M., Wozel G. et al. Efficacy and safety of infliximab vs. methotrexate in patients with moderate-to-severe plaque psoriasis: results of an open-label, active-controlled, randomized trial (RESTORE1). Br J Dermatol 2011 Nov; 165 (5): 1109_-17.

22. Saurat J. H., Stingl G., Dubertret L. et al. Efficacy and safety results from the randomized controlled comparative study of adalimumab vs. methotrexate vs. placebo in patients with psoriasis (CHAMPION). Br J Dermatol 2008 Mar; 158 (3): 558-66.

23. Hassan W. Methotrexate and liver toxicity: role of surveillance liver biopsy. Conflict between guidelines for rheumatologists and dermatologists. Ann Rheum Dis. 1996 May; 55 (5): 273—5.

24. Gossec L., Smolen J. S., Ramiro S. et al. European League Against Rheumatism (EULAR) recommendations for the management of psoriatic arthritis with pharmacological therapies: 2015 update. Ann Rheum Dis 2015; 0:1-12.

25. Coates L. C., Kavanaugh A., Mease P.J. et al. Group for Research and Assessment of Psoriasis and Psoriatic Arthritis (GRAPPA): Treatment Recommendations for Psoriatic Arthritis 2015 [abstract]. Arthritis Rheumatol. 2015; 67 (Suppl 10).

26. Warren R. B., Mrowietz U., von Kiedrowski R. et al. An intensified dosing schedule of subcutaneous methotrexate in patients with moderate to severe plaque-type psoriasis (METOP): a 52 week, multicentre, randomised, double-blind, placebo-controlled, phase 3 trial. Lancet. 2016 Dec 21. pii: S0140-6736 (16) 32127-4.

27. Nestle F. O., Kaplan D. H., Barker J. Psoriasis. N Engl J Med. 2009 Jul 30; 361 (5): 496-509.

28. Kim J., Krueger J. G. The immunopathogenesis of psoriasis. Dermatol Clin. 2015 Jan; $33(1): 13-23$.
29. Warren R. B., Grifiths C. E. M. Systemic therapies for psoriasis: methotrexate, retinoids, and cyclosporine. Clin Dermatol. 2008; 26: 438 — 47.

30. Genestier L., Paillot R., Fournel S. et al. Immunosuppressive properties of methotrexate: apoptosis and clonal deletion of activated peripheral T cells. J Clin Invest. 1998; 102: 322-8.

31. Torres-Álvarez B., Castanedo-Cazares J. P., Fuentes-Ahumada C., Moncada B. The effect of methotrexate on the expression of cell adhesion molecules and activation molecule CD69 in psoriasis. J Eur Acad Dermatol Venereol. 2007; 21: 334-9.

32. Sigmundsdottir H., Johnston A., Gudjonsson J. E. et al. Methotrexate markedly reduces the expression of vascular E-selectin, cutaneous lymphocyte-associated antigen and the numbers of mononuclear leucocytes in psoriatic skin. Exp Dermatol. 2004; 13: 426-34.

33. Cronstein B. N., Naime D., Ostad E. The antiinlammatory effects of methotrexate are mediated by adenosine. Adv Exp Med. 1994; 370: $411-6$.

34. Johnston A., Gudjonsson J. E., Sigmundsdottir $H$. et al. The anti-inlammatory action of methotrexate is not mediated by lymphocyte apoptosis, but by the suppression of activation and adhesion molecules. Clin Immunol. 2005; 114: 154-63.

35. Gladman D. D. Should methotrexate remain the first-line drug for psoriasis? Lancet. 2016 Dec 21. pii: S0140-6736 (16) 32574-0.

36. WHO. Global report on psoriasis. 2016. http:// apps.who.int/iris/ bitstream/10665/204417 ///9789241565189_eng.pdf?ua=I (accessed April 1, 2016).

об авторе:

А.Л. Бакулев - д.м.н., профессор, профрессор кафедры кожных и венерических болезней ФГБОУ ВО «Саратовский ГМУ им. В. И. Разумовского» Минздрава России

\section{Конфликт интересов}

Автор заявляет об отсутствии потенциального конфрликта интересов, требующего раскрытия в данной статье 